\title{
A SUPERREFLEXIVE BANACH SPACE WHICH DOES NOT ADMIT COMPLEX STRUCTURE
}

\author{
STANISLAW J. SZAREK ${ }^{1}$
}

\begin{abstract}
We construct an infinite-dimensional superreflexive real Banach space which does not admit complex structure and consequently is not isomorphic to the Cartesian square of any Banach space. We also construct a variant of Bourgain's example of a complex Banach space with nonunique complex structure and state a number of open problems about structure of Banach spaces and their linear groups.
\end{abstract}

1. Introduction and main results. The main result of this paper is the following

THEOREM 1.1. There exists an infinite-dimensional superreflexive real Banach space which does not admit complex structure. Moreover, it can be chosen to be a subspace of a Banach lattice, which is 2-convex and $q$-concave for any $q>2$ (resp. 2 -concave and $p$-convex for any $p<2$ ).

We have the immediate

COROLLARY 1.2. There exists an infinite-dimensional superreflexive real $B a$ nach space which is not isomorphic to the Cartesian square of any Banach space.

It should be observed that the James' space $J[6]$ constitutes a nonreflexive example for the main statements of Theorem 1.1 and Corollary 1.2. Also, examples of superreflexive spaces which are not isomorphic to their Cartesian squares have been known for some time (see $[3, \mathbf{1}]$ ). However, the space from $[3]$ is known to be isomorphic to some Cartesian square (see also Problem 7.4).

The proof of Theorem 1.1 is based on the following finite-dimensional result:

Proposition 1.3. Given $q \in[2, \infty]$ and $n \in \mathbf{N}$ there exists $Y=Y_{q}^{n} \subset L_{q}$ with $\operatorname{dim} Y=n$ such that if $Z=Y \oplus_{2} H$ ( $H$ any Hilbert space $)$ and if $A: Z \rightarrow Z$ satisfies $A^{2}=-I$, then $\|A\| \geq c_{0} n^{(1 / 2-1 / q) / 2}$, where $c_{0}$ is a numerical constant.

Proposition 1.3 is proved using the results from [19], in turn influenced by [5 and 18]. The procedure of "glueing" finite-dimensional spaces into an infinitedimensional one is taken from [2]; similar schemes were previously employed e.g. in $[3,7$ or 8$]$.

The paper is organized as follows: $\S 2$ explains notation and terminology. $\S 3$ presents known results and preliminary lemmas. $\S 4$ contains the proof of Proposition 1.3. In $\S 5$ we derive Theorem 1.1 from Proposition 1.3. In $\S 6$ we give a sketch

Received by the editors March 15, 1985.

1980 Mathematics Subject Classification (1985 Revision). Primary 46B20; Secondary 46B10, 47D15, 52A22.

${ }^{1}$ Supported in part by NSF grant DMS-8401906. 
of an alternate proof of the result of J. Bourgain [2] on nonuniqueness of complex structure in normed spaces. $\S 7$ lists open problems.

ACKNOWLEDGMENTS. The author would like to thank Haskell Rosenthal for pointing out that the problems considered here were open.

2. Notation and terminology. We use standard Banach space notation, as can be found e.g. in [11]. If $B$ is a measurable subset of $\mathbf{R}^{n}$, we will denote by $\operatorname{vol}(B)$ the usual $n$-dimensional Lebesgue measure of $B$ and by $\operatorname{ac}(B)$ the absolute convex hull of $B$. If $X$ is a normed space, we will denote by $\|\cdot\|_{X}$ its norm; by $L(X)$ the space of bounded linear operators on $X$, endowed with the usual operator norm $\|\cdot\|_{L(X)}$ (which we may also denote by $\|\cdot: X \rightarrow X\|$ ); similarly $L(X, Y) . L_{\mathbf{R}}(X)$ is the space of $\mathbf{R}$-linear operators on the (possibly complex) space $X . B(X)$ is the unit ball of $X ; B\left(l_{p}^{n}\right)=B_{p}^{n}$. If $\left(X_{j}\right)$ is a sequence of normed spaces, $\left(\bigoplus_{j} X_{j}\right)_{l_{p}}$ is the space of sequences $\left(x_{j}\right), x_{j} \in X_{j}$, for which the norm $\left\|\left(x_{j}\right)\right\|=\left\|\left(\left\|x_{j}\right\|_{X_{j}}\right)\right\|_{l_{p}}$ is finite; we will also denote by $X_{1} \oplus_{p} X_{2}$ the $l_{p}$-sum of just two spaces.

For $T \in L(X, Y), \pi_{p}(T)$ denotes the usual $p$-absolutely summing norm of $T$ (see [11, vol. II, pp. 63-64]). We need to know that if $T \in L(X, Y)$ and $S \in$ $L(Y, Z)$, then $\pi_{p}(T) \leq \min \left\{\|S\| \pi_{p}(T), \pi_{p}(S)\|T\|\right\}$. We say that $X$ satisfies the Grothendieck theorem with constant $C$ iff $\pi_{1}(T) \leq C\|T\|_{L\left(X, l_{2}\right)}$ for $T \in L\left(X, l_{2}\right)$ (note that we always have $\pi_{p}(T) \leq \pi_{1}(T)$ for $p \geq 1$ ). If $H$ is a Hilbert space and $T \in L(H), T$ compact, we denote by $\left(s_{j}(T)\right)_{j=1}^{\operatorname{dim} H}$ the sequence of $s$-numbers of $T$ (i.e. the eigenvalues of $|T| \stackrel{\text { df }}{=}\left(T^{*} T\right)^{1 / 2}$, counted with multiplicity and arranged in the nonincreasing order). $\mathrm{hs}(T)=\left\|\left(s_{j}(T)\right)\right\|_{2}$ is the Hilbert-Schmidt norm of $T$; we have $\mathrm{hs}(T)=\pi_{2}(T)$. Furthermore, if $T \in L(H)$ is compact and $\left(s_{j}(T)\right) \in l_{1}$, we define the quasi-norm

$$
\|T\|_{C_{0}}=\sum_{j} \frac{s_{j}(T)}{1+s_{j}(T)}
$$

$\|\cdot\|_{C_{0}}$ satisfies the triangle inequality, but is not positively homogeneous. For our purposes it is essentially sufficient to know that if $S \in L\left(l_{2}^{n}\right)$ and $\|S\|_{C_{0}} \leq \delta n$, then there exists $F \in l_{2}^{n}$ with $\operatorname{codim} F<\sqrt{\delta} n$ such that $\|S x\|_{2} \leq \sqrt{\delta}\|x\|_{2}$ for $x \in F$. Also let us note that if $s_{k}(S)=a$, then $\|S x\|_{2} \leq a\|x\|_{2}$ on a subspace of codimension $<k$ and $\|S x\|_{2} \geq a\|x\|_{2}$ on a subspace of dimension $\geq k$.

Finally, to avoid unnecessary repetitions, let us agree that the letters $c, c_{1}, c^{\prime}$, etc. will denote universal constants while e.g. $c(\delta)$ will denote a constant depending only on a parameter $\delta$.

3. Known results and preliminary lemmas. The following known result is the starting point of this paper:

THEOREM $3.1[\mathbf{1 9}$, THEOREM 1.5]. Given $\delta \in(0,1]$ and $n \in \mathbf{N}$, there exists a norm $\|\cdot\|_{X_{1}^{n}}$ on $\mathbf{R}^{n}$ satisfying

(a) $X_{1}^{n}$ is isometric to a quotient of $l_{1}^{N}$ for some $N \leq 2 n$,

(b) $\|\cdot\|_{2} \leq\|\cdot\|_{X_{1}^{n}} \leq\|\cdot\|_{1}$,

(c) $X_{1}^{n}$ satisfies the Grothendieck theorem with constant $c_{1}$,

(d) if $\|T\|_{L\left(X_{1}^{n}\right)} \leq c(\delta) \sqrt{n}$, then $\|T-\lambda I\|_{C_{0}} \leq \delta n$ for some $\lambda \in \mathbf{R}$. 
REMARK 3.2. Conditions (a) and (b) are clearly equivalent to

$$
\left\{\begin{array}{l}
B\left(X_{1}^{n}\right)=\operatorname{ac}\left\{e_{1}, e_{2}, \ldots, e_{n}, x_{n+1}, \ldots, x_{N}\right\}, \text { where }\left(e_{j}\right) \text { is the } \\
\text { standard unit vector basis and }\left\|x_{j}\right\|_{2} \leq 1 \text { for } n<j \leq N .
\end{array}\right.
$$

In particular, it then follows that $\left[\operatorname{vol}\left(B\left(X_{1}^{n}\right)\right) / \operatorname{vol}\left(n^{-1 / 2} B_{2}^{n}\right)\right]^{1 / n} \leq\left(8 e^{3} / \pi\right)^{1 / 2}<$ 7.2 (see e.g. [17, Lemma 6.5]).

REMARK 3.3. The fact that $X_{1}^{n}$ can be chosen to satisfy the Grothendieck theorem with universal constant $c_{1}$ (and also to have cotype 2 constant $\leq c_{1}$ and other properties not used here) follows from the "random" choice of $x_{j}$ 's; the kernel of the quotient map from (a) is then, with large probability, nearly Euclidean (cf. $[19, \S 5(\mathrm{a})$ or 18 , Remark $4.5 ; 9,14])$. In fact for our purposes it is sufficient to use the classical Grothendieck theorem on operators from $l_{1}$ to $l_{2}$ since, as one can show, the quotient map is a "good" isomorphism on some very large (e.g. $.9 n$-dimensional) "nearly" Euclidean subspace of $l_{1}^{N}$.

CoROllary 3.4. Given $p \in[1,2], \delta \in(0,1]$ and $n \in \mathrm{N}$, there exists a norm $\|\cdot\|_{X_{p}^{n}}$ on $\mathbf{R}^{n}$ such that

(i) $X_{p}^{n}$ is isometric to a quotient of $l_{p}^{N}$ with $N \leq 2 n$,

(ii) if $\|T\|_{L\left(X_{p}^{n}\right)} \leq c(\delta) n^{1 / p-1 / 2}$, then $\|T-\lambda I\|_{C_{0}} \leq \delta n$ for some $\lambda \in \mathbf{R}$,

(iii) $n^{1 / p-1 / 2}\|\cdot\|_{2} \geq\|\cdot\|_{X_{p}^{n}}$ (equivalently $n^{1 / 2-1 / p} B_{2}^{n} \subset B\left(X_{p}^{n}\right)$ ),

(iv) $\left[\operatorname{vol}\left(B\left(X_{p}^{n}\right)\right) / \operatorname{vol}\left(n^{1 / 2-1 / p} B_{2}^{n}\right)\right]^{1 / n} \leq c_{2}$,

(v) $X_{p}^{n}$ satisfies the Grothendieck theorem with constant $c_{1} n^{1-1 / p}$.

PROOF. Consider the same quotient space as in Theorem 3.1, but with respect to the $l_{p}^{N}$-norm rather than the $l_{1}^{N}$-norm. Since $\|\cdot\|_{l_{1}^{N}} \geq\|\cdot\|_{l_{p}^{N}} \geq N^{1 / p-1}\|\cdot\|_{l_{1}^{N}}$, we have also

$$
\|\cdot\|_{X_{1}^{n}} \geq\|\cdot\|_{X_{p}^{n}} \geq N^{1 / p-1}\|\cdot\|_{X_{1}^{n}},
$$

and so (ii) and (v) follow. By Remark 3.2, the quotient map sends the standard unit vector basis of $\mathbf{R}^{N}$ onto $e_{1}, e_{2}, \ldots, e_{n}, x_{n+1}, \ldots, x_{N} \in \mathbf{R}^{n}$ and so $B_{p}^{n} \subset B\left(X_{p}^{n}\right)$; thus $\|\cdot\|_{X_{p}^{n}} \leq\|\cdot\|_{l_{p}^{n}}\left(\leq n^{1 / p-1 / 2}\|\cdot\|_{l_{2}^{n}}\right)$; this shows (iii). Finally, (1) implies that $B\left(X_{p}^{n}\right) \subset N^{1-1 / p} B\left(X_{1}^{n}\right)$. Since $N \leq 2 n$, this combined with the second part of Remark 3.1 proves (iv) with $c_{2}=2^{1-1 / p} \cdot 7.2<11$.

REMARK 3.5. In the terminology of [20], (iii) and (iv) mean that the "volume ratio" of $X_{p}^{n}$ with respect to the ellipsoid $n^{1 / 2-1 / p} B_{2}^{n}$ does not exceed $c_{2}$. Hence, by $\left[20,(*)\right.$, p. 368 or 15, Remark 5], there exists a subspace $E$ of $X_{p}^{n}$ with $\operatorname{dim} E \geq \frac{1}{2} n$ such that for $x \in E$

$$
n^{1 / p-1 / 2}\|x\|_{2} \geq\|x\|_{X_{p}^{n}} \geq c_{3} n^{1 / p-1 / 2}\|x\|_{2}
$$

where $c_{3}=\left(4 \pi c_{2}^{2}\right)^{-1}$.

We also need the following

LEMMA 3.6. Let $(H,|\cdot|)$ be an n-dimensional Hilbert space and $D \in L(H)$ be such that, for some $\lambda \in \mathbf{R},\|D-\lambda I\|_{C_{0}} \leq \delta n$. Then there exists a subspace $F$ of $H$ with $\operatorname{codim} F<2 \sqrt{\delta} n$ such that $\left|\left(D^{2}+I\right) x\right| \geq(1-2 \delta)|x|$ for $x \in F$.

PROOF. Let $F_{0} \subset H$ be a subspace with $\operatorname{codim} F_{0}<\sqrt{\delta} n$ such that $|D x-\lambda x| \leq$ $\sqrt{\delta}|x|$ for $x \in F_{0}$. Then $F=F_{0} \cap D^{-1}\left(F_{0}\right)$ is the required subspace. 
4. Proof of Proposition 1.3. By duality, it is enough to prove the assertion of Proposition 1.3 with $Y$ replaced by a quotient of $L_{p}$, where $1 / p=1-1 / q$. Specifically, let us consider $Z=X_{p}^{n} \oplus_{2} H$, where $X_{p}^{n}$ is obtained via Corollary 3.4 applied with $\delta=2^{-6}$. Set $c_{0}=\min \left\{c\left(2^{-6}\right),\left(4 c_{3} c_{1}\right)^{-1 / 2}\right\}$, where $c\left(2^{-6}\right), c_{1}$ and $c_{3}$ come from Corollary 3.4(ii), (v) and (2), respectively. To prove the Proposition let us assume, to the contrary, that there exist $A \in L(Z)$ with $A^{2}=-I$ and $\|A\|<c_{0} n^{(1 / 2-1 / q) / 2}$. Write $A$ as a $2 \times 2$ matrix with operator entries

$$
A=\left[\begin{array}{ll}
A_{11} & A_{12} \\
A_{21} & A_{22}
\end{array}\right] \begin{gathered}
X_{p}^{n} \\
H
\end{gathered} .
$$

Clearly,

$$
\left\|A_{i j}\right\|<c_{0} n^{(1 / 2-1 / q) / 2}=c_{0} n^{(1 / p-1 / 2) / 2}
$$

for $i, j=1,2$ and the corresponding operator norms. In particular, Corollary 3.4(ii) implies that there exists $\lambda \in \mathbf{R}$ such that $\left\|A_{11}-\lambda I_{X_{p}^{n}}\right\|_{C_{0}}<2^{-6} n$ and so, by Lemma 3.6 applied with $D=A_{11}$ and $\delta=2^{-6}$, we get that there exists a subspace $F$ of $X_{p}^{n}$ (identified with $\mathbf{R}^{n}$ ) with $\operatorname{codim} F<n / 4$ such that

$$
\left\|\left(A_{11}^{2}+I\right) x\right\|_{2} \geq\left(1-2^{-5}\right)\|x\|_{2} \text { for } x \in F .
$$

On the other hand, $\left.A^{2}\right|_{X_{p}^{n}}=A_{11}^{2}+A_{12} A_{21}=-I_{X_{p}^{n}}$ and so, denoting $R=$ $-A_{12} A_{21}$, we have

$$
A_{11}^{2}+I_{X_{p}^{n}}=R
$$

By Corollary 3.4(v) and (3),

$$
\pi_{2}(R) \leq\left\|A_{12}\right\| \cdot \pi_{2}\left(A_{21}\right) \leq c_{1} n^{1-1 / p}\left\|A_{12}\right\| \cdot\left\|A_{21}\right\| \leq c_{1} c_{0}^{2} n^{1 / 2} .
$$

Now choose $E_{1} \subset X_{p}^{n}$ with $\operatorname{dim} E_{1} \geq n / 2$ so that (2) holds for $x \in R E_{1}$ ( $E_{1}=$ $R^{-1} E$ with $E$ given by Remark 3.5 works). Of course, the left inequality in (2) holds for all $x \in X_{p}^{n}$ (Corollary 3.4(iii)). It thus follows that $\pi_{2}\left(\left.R\right|_{E_{1}}:\left(E_{1},\|\cdot\|_{2}\right) \rightarrow\right.$ $\left.l_{2}^{n}\right)=\operatorname{hs}\left(\left.R\right|_{E_{1}}\right) \leq c_{3} c_{1} c_{0}^{2} n^{1 / 2} \leq \frac{1}{4} n^{1 / 2}$ (note our choice of $c_{0}$ ). Consequently, there exists a further subspace $E_{2} \subset E_{1}$ with $\operatorname{dim} E_{2} \geq \frac{1}{2} \operatorname{dim} E_{1} \geq \frac{1}{4} n$ such that

$$
\|R x\|_{2} \leq \sqrt{4 / n} \mathrm{hs}\left(\left.R\right|_{E_{1}}\right)\|x\|_{2} \leq \frac{1}{2}\|x\|_{2} \text { for } x \in E_{2} .
$$

This (remember (5)) contradicts (4) and proves Proposition 1.3.

5. Proof of Theorem 1.1. In this section we deduce Theorem 1.1 from Proposition 1.3. The former one will follow immediately from the following

PROPOSITION 5.1. There exist a sequence of integers $\left(n_{k}\right)$ and a sequence of reals $q_{k} \downarrow 2$ such that if $Y=\left(\bigoplus Y_{q_{k}}^{n_{k}}\right)_{l_{2}}$, where the $Y_{q}^{n}$ 's are the spaces given by Proposition 1.3, then there is no operator $A \in L(Y)$ such that $A^{2}=-I$.

PROOF. We follow the argument from [2]. However, since we work with subspaces of $L_{q}$-spaces, we do not have to go through an interpolation argument.

Define sequences $\left(n_{k}\right)$ and $\left(q_{k}\right)$ inductively by

(10) $q_{1}=n_{1}=4$,

$\left(2^{\circ}\right)$ if $\left(q_{j}\right),\left(n_{j}\right)$ are defined for $j<k$, choose $q_{k} \in\left(2, q_{k-1}\right)$ so that 
(i) $n_{k-1}^{1 / 2-1 / q_{k}} \leq 2$

and then choose $n_{k}>n_{k-1}$ so that

(ii) $c_{0} n_{k}^{\left(1 / 2-1 / q_{k}\right) / 2} n_{k-1}^{-1 / 2}>k$,

where $c_{0}$ is the constant from Proposition 1.3.

Observe that since $n_{k} \uparrow \infty$, (i) implies that $q_{k} \downarrow 2$ (we could also require this additionally).

Now suppose that there exists $A \in L(Y)$ with $A^{2}=-I$ and fix $k>\|A\|$. Denote by $P_{j}$ the natural projection of $Y$ onto $Y_{q_{j}}^{n_{j}}$. Set $Z^{\prime}=Y_{q_{k}}^{n_{k}}+A Y_{q_{k}}^{n_{k}}$. Then $A Z^{\prime}=Z^{\prime}$ and $Z^{\prime}=Y_{q_{k}}^{n_{k}} \oplus_{2} F^{\prime}$, where $F^{\prime}=\left(I-P_{k}\right) Z^{\prime}=\left(I-P_{k}\right) A Y_{q_{k}}^{n_{k}}$. Clearly, $\operatorname{dim} F^{\prime} \leq n_{k}$ and $F^{\prime} \subset\left(\bigoplus_{j \neq k} P_{j} F^{\prime}\right)_{l_{2}}$. We have, for $j<k$,

$$
d\left(P_{j} F^{\prime}, l_{2}^{\operatorname{dim} P_{j} F^{\prime}}\right) \leq\left(\operatorname{dim} P_{j} F^{\prime}\right)^{1 / 2} \leq n_{j}^{1 / 2} \leq n_{k-1}^{1 / 2}
$$

similarly, for $j>k$,

$$
d\left(P_{j} F^{\prime}, l_{2}^{\operatorname{dim} P_{j} F^{\prime}}\right) \leq\left(\operatorname{dim} P_{j} F^{\prime}\right)^{1 / 2-1 / q_{j}} \leq n_{k}^{1 / 2-1 / q_{k}} \leq 2
$$

(we used $2^{\circ}(\mathrm{i})$ and $[\mathbf{1 1}]$ ); this shows that

$$
d\left(F^{\prime}, l_{2}^{\operatorname{dim} F^{\prime}}\right) \leq \max \left\{2, n_{k-1}^{1 / 2}\right\}=n_{k-1}^{1 / 2} .
$$

Hence if $Z=Y_{q_{k}}^{n_{k}} \oplus_{2} l_{2}^{\operatorname{dim} F^{\prime}}$, then $d\left(Z, Z^{\prime}\right) \leq n_{k-1}^{1 / 2}$. Combining this with Proposition 1.3 we see that

$$
\left\|\left.A\right|_{Z^{\prime}}\right\|_{L\left(Z^{\prime}\right)} \geq c_{0} n_{k}^{\left(1 / 2-1 / q_{k}\right) / 2} n_{k-1}^{-1 / 2}>k
$$

by $2^{\circ}$ (ii); a contradiction. This proves Proposition 5.1 and concludes the proof of Theorem 1.1.

REMARK 5.2. It is clear from the proof that Proposition 5.1 (and hence also Theorem 1.1 and Corollary 1.2) remains true if we replace $Y$ by $Y \oplus H$, where $H$ is any Hilbert space.

6. A variant of Bourgain's example of a space with nonunique complex structure. In this section we indicate how, arguing analogously as in $\S \S 3-5$, one can reprove the following results of J. Bourgain [2].

THEOREM 6.1. There exists a (superreflexive) complex Banach space $X$ such that if one defines new multiplication by complex scalars by $\lambda \odot x=\bar{\lambda} x$ and denotes the resulting new complex Banach space by $\bar{X}$, then $X$ and $\bar{X}$ are not isomorphic. As a consequence, there exists a real Banach space, which admits two nonisomorphic complex structures.

Similarly as in [2], the argument is based on some finite-dimensional fact (slightly stronger than the corresponding result from [2]).

Proposition 6.2. Given $q \in[2, \infty]$ and $n \in \mathbf{N}$ there exists (a complex Banach space) $Y=\tilde{Y}_{q}^{n} \subset L_{q}$ with $\operatorname{dim} Y=n$ such that if $Z=Y \oplus_{2} H$ ( $H$ any complex Hilbert space) and $T: Z \rightarrow Z$ is invertible and complex antilinear (i.e. $T$ is $\mathbf{R}$-linear and $T(\lambda x)=\bar{\lambda} T x$ for $\lambda \in \mathbf{C}$ and $x \in Z)$, then $\|T\| \cdot\left\|T^{-1}\right\| \geq c_{0}^{\prime} n^{1 / 2-1 / q}$.

Once this is shown, we argue exactly as in [2] (and very similarly as in $§ 3$ of this paper, Proposition 6.2 playing the role of Proposition 1.3) to prove that $X=\left(\bigoplus \tilde{Y}_{q_{k}}^{n_{k}}\right)_{l_{2}}$ verifies Theorem 6.1 for the appropriate choice of $\left(n_{k}\right)$ and $\left(q_{k}\right)$. 
Also the (nearly "soft") proof of Proposition 6.2 goes along similar lines as the proof of Proposition 1.3. First [19, Lemma 3.5A, Theorem 1.4, cf. $\S 5(\mathrm{a})]$ readily imply (cf. the proof of Corollary 3.4) the existence, for given $p \in[1,2], \delta \in(0,1]$ and $n \in \mathbf{N}$, of a (complex) norm $\|\cdot\|_{\tilde{X}_{p}^{n}}$ on $\mathbf{C}^{n}$ satisfying conditions (i), (iii), (iv) and (v) of Corollary $3.4((\mathrm{v})$ for R-linear operators) and additionally

$$
\left\{\begin{array}{l}
\text { if } S: \mathbf{C}^{n} \rightarrow \mathbf{C}^{n} \text { is } \mathbf{C} \text {-antilinear and at least } r \geq \delta n s \text {-numbers } \\
\text { of } S \text { are } \geq \alpha, \text { then }\|S\|_{L_{\mathbf{R}}\left(\tilde{X}_{p}^{n}\right)} \geq c^{\prime}(\delta) \cdot \alpha \cdot n^{(1 / p-1 / 2) / 2},
\end{array}\right.
$$

where, for the purpose of determining $s$-numbers, $S$ is thought of as $\mathbf{R}$-linear (note here that in the language of [19] $S$ is $\mathrm{C}$-antilinear iff it is R-linear and $S A=-A S$, where $A: \mathbf{C}^{n} \rightarrow \mathbf{C}^{n}$ is given by $\left.A x=i x\right)$.

By duality, it is again enough to show that the assertion of Proposition 6.2 holds with $Z$ replaced by $Z^{\prime}=\tilde{X}_{p}^{n} \oplus_{2} H$, where $\tilde{X}_{p}^{n}$ is obtained as described above (with $\delta=1 / 2$ ) and $1 / p=1-1 / q$. To this end, let $T: Z^{\prime} \rightarrow Z^{\prime}$ be $\mathbf{C}$-antilinear and invertible and, to argue by contradiction, suppose that

$$
\|T\| \cdot\left\|T^{-1}\right\|<\min \left\{\frac{1}{2}\left(c^{\prime}\left(\frac{1}{2}\right)\right)^{2}, c^{\prime \prime}\right\} n^{1 / p-1 / 2},
$$

where the choice of $c^{\prime \prime}$ will be indicated later (note that $1 / p-1 / 2=1 / 2-1 / q$ ). Consider the matrix representations

$$
T=\left[\begin{array}{ll}
T_{11} & T_{12} \\
T_{21} & T_{22}
\end{array}\right] \begin{gathered}
\tilde{X}_{p}^{n} \\
H
\end{gathered}, \quad T^{-1}=\left[\begin{array}{ll}
T_{11}^{\prime} & T_{12}^{\prime} \\
T_{21}^{\prime} & T_{22}^{\prime}
\end{array}\right] \begin{gathered}
\tilde{X}_{p}^{n} \\
H
\end{gathered}
$$

Denote by $k$ the smallest integer $\geq n / 2$ and set $a=s_{k}\left(T_{11}\right), a^{\prime}=s_{k}\left(T_{11}^{\prime}\right)$. Then, by (ii)' and (7),

$$
a a^{\prime}<1 / 2 \text {. }
$$

On the other hand (cf. the remark at the end of §2), there exists (an R-linear subspace) $F \subset \tilde{X}_{p}^{n}, \operatorname{codim} F=k-1<n / 2\left(\right.$ resp. $\left.F^{\prime} \subset \tilde{X}_{p}^{n}\right)$ such that

$$
\left\|T_{11} x\right\|_{2} \leq a\|x\|_{2} \quad \text { for } x \in F
$$

and, respectively,

$$
\left\|T_{11}^{\prime} x\right\|_{2} \leq a^{\prime}\|x\|_{2} \quad \text { for } x \in F^{\prime}
$$

Now observe that

$$
I_{\tilde{X}_{p}^{n}}=T_{11} T_{11}^{\prime}+T_{12} T_{21}^{\prime}
$$

and, similarly as in the argument leading to (6), choose a (real or complex) subspace $E_{1}$ of $\tilde{X}_{p}^{n}$ with (real) $\operatorname{dim} E_{1} \geq \frac{3}{2} n$ such that $T_{12} T_{21}^{\prime} E_{1}$ is "nearly" Euclidean in the sense of (2) (we use our analogues of (iii) and (iv) from Corollary 3.4 and [15, Théorème 8] (or [16, Remark 2 or 17, Proposition 3]). Then $h s\left(\left.T_{12} T_{21}^{\prime}\right|_{E_{1}}\right) \leq \frac{1}{4} n^{1 / 2}$ if $c^{\prime \prime}$ in (7) was chosen small enough. Accordingly, there exists a further (real) subspace $E_{2} \subset E_{1}, \operatorname{dim} E_{2} \geq n$, such that, for $x \in E_{2}$,

$$
\left\|T_{12} T_{21}^{\prime} x\right\|_{2} \leq \sqrt{\frac{2}{n}} \cdot \frac{1}{4} n^{1 / 2}\|x\|_{2}=\frac{\sqrt{2}}{4}\|x\|_{2} .
$$


This, combined with (8), (9), (9)' and (10), shows that if $x \in E_{2} \cap F^{\prime} \cap T_{11}^{-1}(F)$ (a nontrivial subspace), then

$$
\|x\|_{2}=\left\|T_{11} T_{11}^{\prime} x+T_{12} T_{21}^{\prime} x\right\|_{2} \leq\left(a a^{\prime}+\frac{\sqrt{2}}{4}\right)\|x\|_{2} \leq\left(\frac{1}{2}+\frac{\sqrt{2}}{4}\right)\|x\|_{2},
$$

a contradiction if $x \neq 0$. This shows Proposition 6.2 and concludes the proof of Theorem 6.1.

REMARK 6.3. As was communicated to the author by N. J. Kalton, the example [8] of a (closed) subspace of $L_{p}(p \in[0,1))$, every endomorphism in which is a multiple of the identity, can be modified to yield a like complex space such that every $\mathbf{R}$-linear endomorphism in it is a (complex) multiple of the identity. It can also be modified to yield the isometric version of Theorem 6.1.

\section{Open problems.}

Problem 7.1. Let $X$ be a (real) Banach lattice (resp. a space with unconditional basis). Does $X$ admit complex structure? Is $X$ isomorphic to $Y^{2}$ for some Banach space $Y$ ? Lattice isomorphic to $Y^{2}$ for some Banach lattice $Y$ ? It should be noticed that the spaces constructed in this paper do not even have the so-called local unconditional structure (see [4]).

Problem 7.2. Does there exist a real Banach space nonisomorphic to a Hilbert space which admits unique complex structure?

Problem 7.3. In [19] the "worst" possible finite-dimensional examples for nonexistence (resp. nonuniqueness) of complex structure were given. Under what nontrivial assumptions do we have positive results? In particular, if a space has a 1-unconditional basis?

Problem 7.4. Can we replace in Corollary 1.2 " $X \not Y^{2}$ " by " $X \not Y^{m}$ " for given $m>2$ ? Any $m>2$ ?

Problem 7.5. Under what general assumptions about a group of operators on $l_{2}^{n}$, does some element of the group not admit "good" approximation by a multiple of identity in the quasi-norm $\|\cdot\|_{C_{0}}$ (equivalently, satisfies the condition $\left(M_{k, \alpha}\right)$ from [19] for large $k, \alpha)$ ? For groups acting irreducibly on $l_{2}^{n}$ this was essentially done in [12]; [19] provides other examples. Similarly, under what general assumptions about a group $\Gamma$ does there exist a Banach space $X$ such that there are no representations of $\Gamma$ acting on $X$ ? Note that the assertion of Theorem 1.1 from this paper can be restated as follows. "There is no representation (of the circle group) $\sigma: S^{1} \rightarrow L(X)$ with $\sigma(-1)=-I$ " and one can ask under what general assumptions on a group (resp. representation) a statement like this is possible (this may be related to $[\mathbf{1 3}])$. Also recall the well-known question about existence of a Banach space, all bounded linear operators on which one of the form $\lambda I+\Delta$ with $\Delta$ compact (resp. nuclear) (cf. Remark 6.3 and [8]). This seems to be quite hard; one can ask instead for an example of a space $X$ for which the quotient algebra of $L(X)$ by the ideal of compact (resp. nuclear) operators is "small".

Problem 7.6. Do we have a complex version of Corollary 1.2? It would presumably follow if we had a complex version of Theorem 1.5 from [19].

\section{REFERENCES}

1. S. F. Bellenot, Tsirelson superspaces and $l_{p}$, Proc. Amer. Math. Soc. 94 (1985).

2. J. Bourgain, A complex Banach space such that $X$ and $\bar{X}$ are not isomorphic, preprint. 
3. T. Figiel, An example of infinite dimensional reflexive Banach space non-isomorphic to its Cartesian square, Studia Math. 42 (1972), 295-306.

4. T. Figiel, S. Kwapien and A. Pelczynski, Sharp estimates for the constants of local unconditional structure for Minkowski spaces, Bull. Acad. Polon. Sci. Ser. Sci. Math. 25 (1977), 1221-1226.

5. E. D. Gluskin, Finite dimensional analogues of spaces without a basis, Dokl. Akad. Nauk SSSR 261 (1981), 1046-1050. (Russian)

6. R. C. James, Bases and reflexivity of Banach spaces, Ann. of Math. 52 (1950), 518-527.

7. W. B. Johnson, Banach spaces all of whose subspaces have the approximation property, Special Topics of Applied Math. (Proc. Sem. Ges. Math. Datenverarb., Bonn, 1979), NorthHolland, Amsterdam, 1980, pp. 15-26.

8. N. J. Kalton and J. W. Roberts, A rigid subspace of $L_{0}$, Trans. Amer. Math. Soc. 266 (1981), 645-654.

9. S. V. Kisliakov, On space with "small" annihilators, Zap. Nauchn. Sem. Leningrad Otdel. Mat. Inst. Steklov 65 (1976), 192-195. (Russian)

10. D. R. Lewis, Finite dimensional subspaces of $L_{p}$, Studia Math. 63 (1978), 207-212.

11. J. Lindenstrauss and L. Tzafriri, Classical Banach spaces, Vols. I and II, Springer-Verlag, Berlin and New York, 1977, 1979.

12. P. Mankiewicz, Finite dimensional Banach spaces with symmetry constant of order $\sqrt{n}$, Studia Math. 79 (1984), 193-200.

13. B. S. Mitiagin, The homotopy structure of the linear group of a Banach space, Uspekhi Mat. Nauk 25 (1970), 63-107. (Russian)

14. G. Pisier, Une nouvelle classe d'espaces vérifiant le théorème de Grothendieck, Ann. Inst. Fourier (Grenoble) 28 (1978), 69-90.

15. M. Rogalski, Sur le quotient volumique d'un espace de dimension finie, Séminaire Initiation Analyse 1980/81, Comm. No. 3, Univ. Paris VI.

16. S. J. Szarek, On Kashin's almost orthogonal decompositon of $l_{1}^{n}$, Bull. Acad. Polon. Sci. 26 (1978), 691-694.

17. __ Volume estimates and nearly Euclidean decompositions for normed spaces, Séminaire Analyse Fonctionnelle 1979-1980, Exp. No. 25, Ecole Polytechnique, Palaiseau.

18. __, The finite dimensional basis problem with an appendix on nets of Grassmann manifolds, Acta Math. 151 (1983), 153-179.

19. __ On the existence and uniqueness of complex structure and spaces with "few" operators, Trans. Amer. Math. Soc. 293 (1986), 339-353.

20. S. J. Szarek and N. Tomczak-Jaegermann, On nearly Euclidean decompositions for some classes of Banach spaces, Compositio Math. 40 (1980), 367-385.

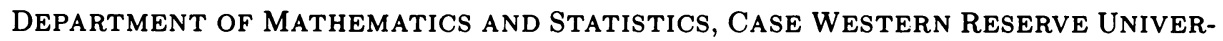
Sity, CleVEland, OHIO 44106 